\title{
On the question of the relationship of variations in geophysical fields, lunar-solar tidal effects and seismic events
}

\author{
Elena Bataleva* \\ Research Station of the Russian Academy of Sciences in Bishkek, 720049, Bishkek, Kyrgyzstan
}

\begin{abstract}
Based on the results of instrumental observations made in the Tien Shan, the interrelation of time variations of a number of geophysical fields and seismic events is established. According to the results of the correlation analysis, it was concluded that the fault zones are characterized by significantly higher variations of geophysical fields, an intense response to weak external influences in the form of a solid moon-solar tide.
\end{abstract}

\section{Introduction}

This paper discusses issues related to the development of new approaches to the study of physical field variations caused by deformation processes occurring in the Earth's crust. The study of electromagnetic phenomena caused by modern geodynamic processes occurring immediately before seismic events is carried out using monitoring observations. One of the tasks of geophysical monitoring is the identification of variations in electromagnetic fields associated with pre-seismic phenomena, which is due to existing ideas about the close relationship of seismicity with changes in the fluid regime and the nature of the fractures of the Earth's crust. Since 2003, monitoring studies of electromagnetic parameters by the magnetotelluric sounding method (MTS) (Fig. 1) have been carried out on the territory of the Bishkek Proving Ground (BPG). The impetus for the development of research was the Kambarata experiment, which confirmed the relationship between changes in the stress-strain state of the Earth's crust blocks and variations in electrical conductivity $[1,2]$.

\section{Azimuthal magnetotelluric monitoring technique}

The uniqueness of the MTS method among electrical prospecting methods is due to the fact that the response to the excitation of the geological environment is presented in the form of the impedance tensor $\mathrm{Z}$, which reflects the relationship of the horizontal components of the electric and magnetic fields. This transfer function carries information about the patterns of distribution of geoelectric parameters of the medium for all directions.

*Corresponding author: elena.bataleva@gmail.com 


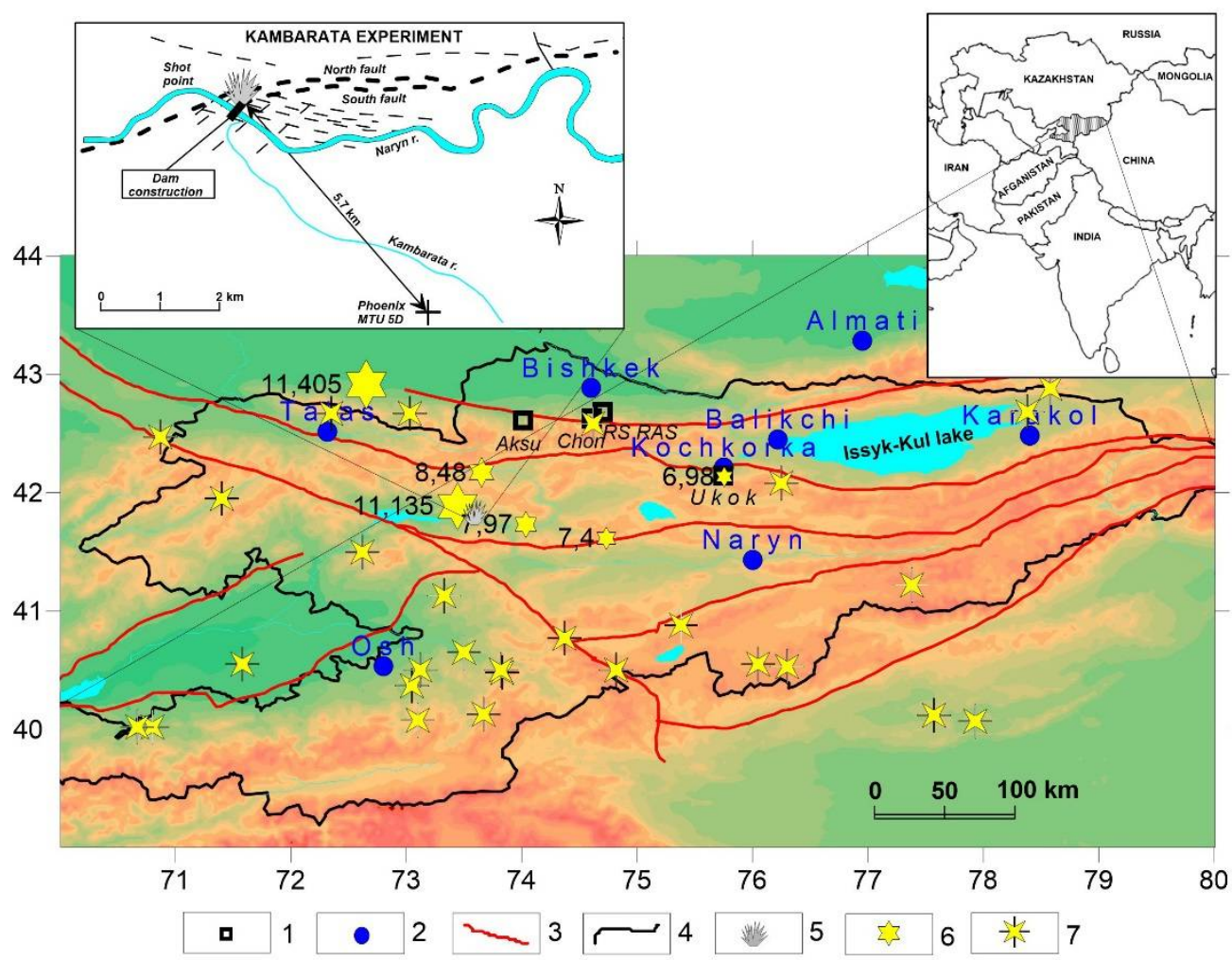

Fig. 1. Location map of Kambarata shot, of stationary points of the MT-monitoring and point DMT Ukok; performed on the territory of the Central Tien Shan: 1 - stationary points of MT-monitoring and point DMT Ukok; 2 - main settlements; 3 - regional faults; 4 - border of Kyrgyzstan; 5 Kambarata shot; 6 - seismic events that occurred during the registration in the area of Kambarata shot; 7 - seismic events that occurred during the registration at the point of DMT Ukok.

When conducting monitoring studies, a very high accuracy of determination of the main $\left(Z_{x y}, Z_{y x}\right)$ and additional $\left(Z_{x x}, Z_{y y}\right)$ components of the impedance tensor must be ensured. However, when determining the components of the impedance tensor, one has to deal with such a complicating factor as the influence of noise distorting the useful signal. Traditionally, various methods of statistical processing are used to eliminate industrial interference and the influence of near-surface heterogeneities. However, to improve the quality of the MTS data distorted by the influence of interference, we used the amplitudephase correction technique [3] when correcting the main and additional impedances. The processed data are used to construct time-frequency series according to the azimuthal magnetotelluric (MT) monitoring technique. When analyzing the behavior of the additional impedance, it is necessary to take into account that according to the methodology "for part of the frequency range the phase and phase curves calculated from the amplitude curves can correspond to each other with a shift that is a multiple of $90^{\circ}$. The processed data are used to construct time-frequency series according to the azimuthal monitoring technique. The technique consists in the following: (1) simultaneous consideration of the dependences of the variations of all components of the impedance tensor (apparent resistance, impedance phase, real and imaginary parts of the main, additional impedance and tipper (hereinafter electromagnetic parameters)) immediately on three parameters - time, sounding period and azimuth (Fig. 2, 3); (2) correlation between tidal influences and changes in electromagnetic 
parameters [4-6], which serve as a criterion for assessing the sensitivity of sensing points, are clearly presented in the form of multidimensional correlation polar diagrams (Fig. 4, 5).
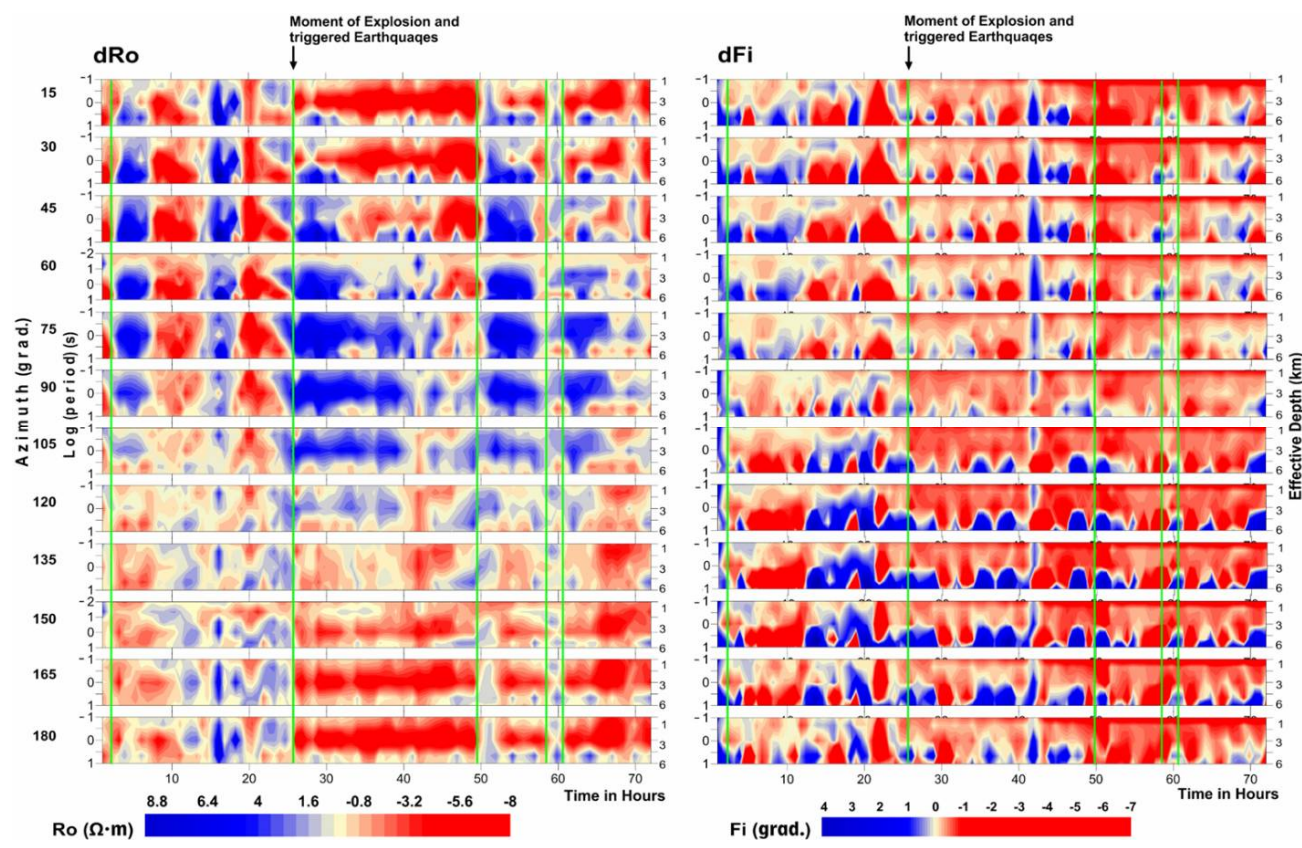

Fig. 2. Frequency-time series of azimuthal MT monitoring of variations in apparent electrical resistance and impedance phases recorded during the Kambarata experiment (12.22.2009). The green lines indicate earthquakes (Fig. 1).
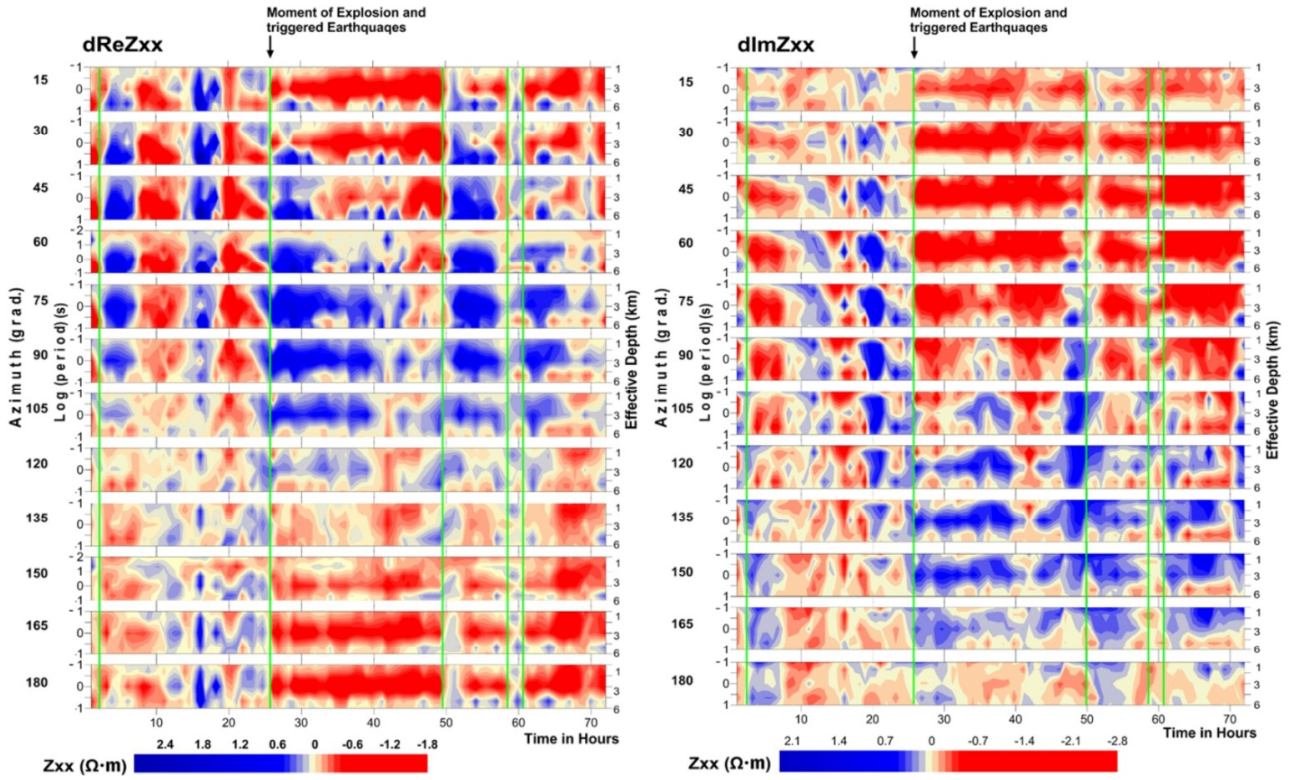

Fig.3. Time-frequency series of azimuthal MT monitoring of the variations of the real and imaginary parts of the additional impedance $Z_{x x}$, recorded during the Kambarata experiment (12.22.2009). The green lines indicate earthquakes (Fig. 1). 


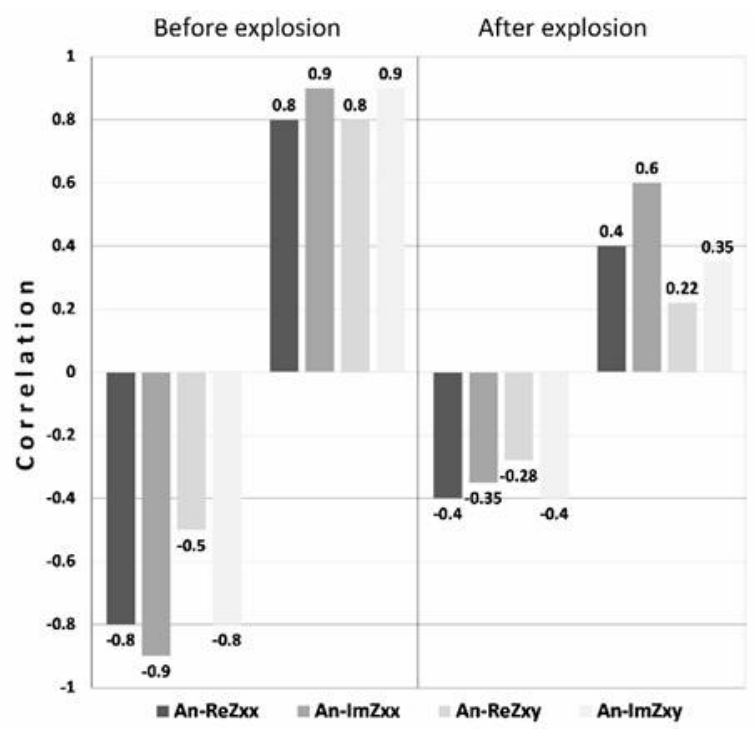

Fig. 4. Change in the correlation between the vertical component of tidal impacts and variations in electromagnetic parameters before and after the Kambarata explosion (12.22.2009).

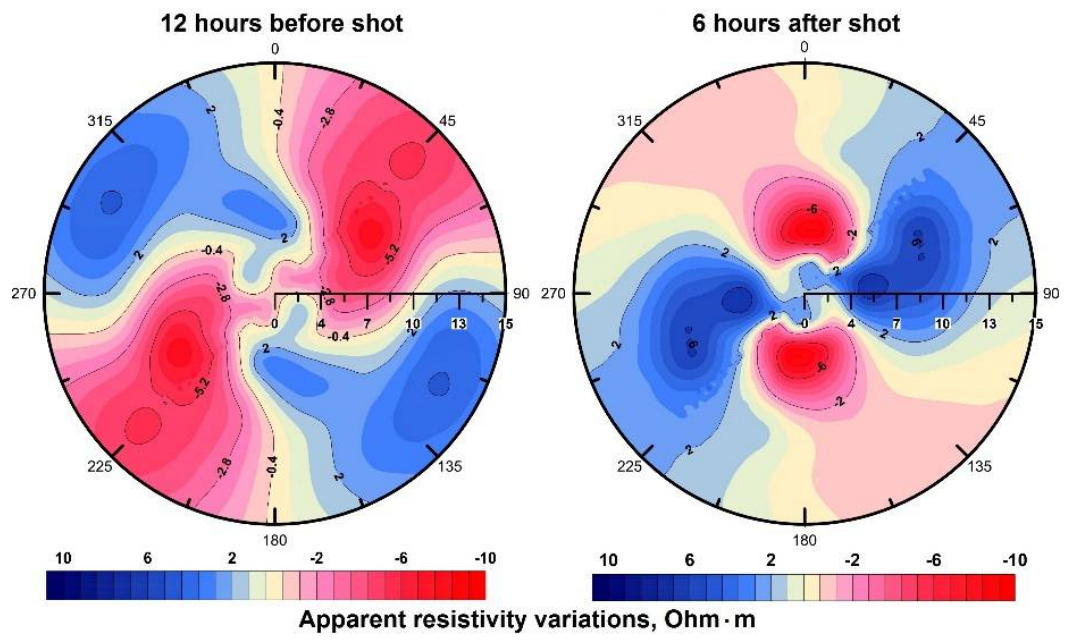

Fig. 5. Correlation polar diagrams for the observation point of deep MTS in the area of the Kambarata explosion of variations in apparent resistance 12 hours before the explosion and 6 hours after the explosion (the root from the sounding period is plotted along the radius axis).

The use of the azimuthal MT monitoring technique confirmed the relationship between changes in the stress-strain state of the Earth's crust blocks and variations in electrical conductivity in accordance with the accepted concept $[4,7]$ of fluid redistribution in the porous-fracture space of rocks caused by deformations.

\section{Results of azimuthal MT-monitoring}

Figures 2 and 3 show the results of applying the azimuthal MT monitoring technique for constructing frequency-time series (FTS), which clearly reflect the moment of the 
Kambarata explosion, the seismic events initiated by it, and changes in apparent resistance in orthogonal directions. The Kambarata experiment (December 22, 2009) included seismological, electromagnetic and GPS observations made by the Research Station RAS (Fig. 1). The power of an industrial explosion was $2.8 \mathrm{Kt}$ in TNT equivalent. The distance from the blast point to the magnetotelluric station Phoenix MTU 5D was $5.7 \mathrm{~km}$. The data obtained as a result of the seismic observations made it possible to establish the presence of shifts due to the explosion along the fault adjacent to the explosion point, and also to record a series of induced weak seismic events at a distance of up to $16 \mathrm{~km}$ from the explosion point 12 seconds after the explosion.

In a detailed analysis of the behavior of all electromagnetic parameters, the following is noteworthy: (1) changes in the parameter occurring in the orthogonal directions are more clearly reflected in the FTS of variations in apparent resistance than in the FTS of impedance phases; (2) the confinement of seismic events to areas with high gradients of variations in electromagnetic parameters is more pronounced in the FTS of variations in apparent resistance; (3) based on the correlation analysis of the FTS components of the impedance tensor components with time series of tidal effects, a high information content of imaginary components and additional impedances for MT monitoring was established.

To calculate the components of tidal effects, we use the Tide.exe program, a theoretical calculation of the tidal deformation of the planet's surface, in which it is based on the fact that the tidal potential is represented as the sum of spherical waves expressed through Legendre polynomials. Each wave has a constant period and amplitude, and the phase depends on the local latitude and the current ephemeris of space bodies. Used decomposition H.G. Wenzel (1996) for a theoretical tide includes more than 1000 waves. Then, theoretical calculations are verified by observation using a Scintrex CG-5 Autograf gravimeter, which is installed in an adit with a constant temperature of +8 deg $\mathrm{C}$ in the territory of the Research Station RAS (30 km from the city). The observations are carried out with an interval of $12 \mathrm{~s}$, and then during processing they are averaged with the interval that is necessary for the tasks and the required data discretization. It should be noted the very high quality of the observed data (about $0.001 \mathrm{mGal}$ ). In this case, interferences are seismic events that are clearly recorded by a gravimeter (Golitsin pendulum). Figure 4 shows the results of evaluating the correlation coefficients of variations in the real and imaginary components of the main and additional impedance with lunar-solar tidal effects and changes in correlation relationships. The low level of correlation coefficients after the explosion indicates that, in addition to tidal influences, tectonic sources of changes in the stress-strain state of the Earth's crust blocks are actively involved in the formation of electrical resistance variations. The results of variations in the apparent resistance before and after the explosion in the form of correlation polar diagrams are shown in Figure 5. The diagrams reflect the parameter changes occurring in orthogonal directions.

Estimates of the correlation coefficients of variations in the apparent resistance modulus and impedance phases with lunar-solar tidal influences are performed for all monitoring points on the territory of the BPG. An example of a comparative analysis of the dynamics of the behavior of the parameters of the electromagnetic field and lunar-solar tides is shown in Figures 6 and 7.

Using correlation analysis of FTS of electromagnetic parameters, indicator strainsensitive elements of the geomedium are identified that correspond to zones of dynamic influence of active faults in the Northern Tien Shan. The strain sensitivity of rock masses is estimated by the level of correlation between gravitational tidal influences and variations of electromagnetic parameters. The orientation of the stable correlation clusters in the polar correlation diagrams corresponds to the strike of fault faults, which indicates the strainsensitive nature of the zones of dynamic influence of active faults in the Northern Tien Shan [8]. As a result of the analysis of the electromagnetic parameters of the environment 
for the relationship with the lunar-solar tidal effects, it was found that the correlation dependences are most pronounced in changes in the real and imaginary parts of the additional impedances than the main ones. Variations of the reactive component of electrical resistance associated with changes in capacitive and inductive characteristics of rock masses during deformation processes respond to tidal influences and deformations no less actively than variations of the active component of electrical resistance.

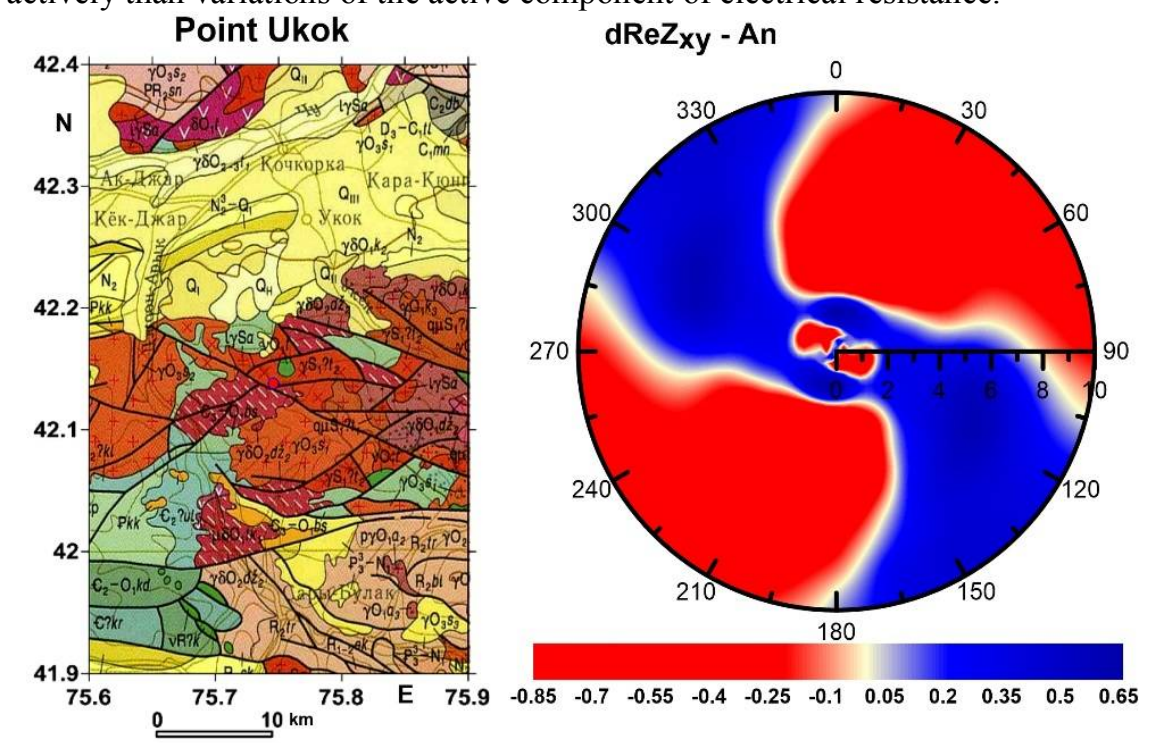

Fig. 6. Correlation polar diagrams for the regime point of deep MTS Ukok (Fig. 1), (ReZxy - An) correlation of the NWR of the variations of the real part of the main impedance and the vertical component of the lunar-solar tidal influences (the root from the sounding period is plotted along the radius axis, coefficient values correlations are shown in contours).

It has been established that under the influence of lunar-solar tides, maximum variations in apparent resistance occur in strain-sensitive zones confined to fault structures $[2,9]$. As in FTSs constructed for the Kambarata explosion, a characteristic feature of the revealed anomalies in FTSs constructed for the Ukok security point is a change in apparent resistance in orthogonal directions, which corresponds to the model of fluid redistribution in the pore-fractured rock space [4, 7]. This model was confirmed when conducting monitoring studies both at stationary points and at sensitive and specialized points of MT monitoring of the Tien Shan.

Figure 7 shows the results of a field experiment carried out in 2018 at the Ukok MT monitoring site in the Kochkor Basin (Fig. 1), as a result of which it was established that there is a stable relationship between the anisotropy of electrical resistance and the energy characteristic of an electromagnetic field of endogenous origin. When comparing the obtained parameters with the distribution of seismicity, attention is drawn to the fact that the seismic events are confined to areas with high gradients of apparent resistance variations and areas of decline in energy characteristics.

\section{Conclusions}

Field experiments have confirmed the concept of the relationship between variations in apparent resistance, lunar-solar tidal effects and the stress-strain state of the medium. The performed studies indicate the need to take into account the peculiarities of the tectonic structure of the study area [8], for which an analysis of the relationship of geodynamic 
processes, apparent resistance and lunar-solar tidal influences is performed. Under the influence of lunar-solar tides, the maximum variations in apparent resistance occur in strain-sensitive zones confined to fault structures, the strike of which is reflected in the correlation polar diagrams. It is noteworthy that the seismic events are confined to areas with high gradients of variations in the apparent resistance and impedance phase, i.e. time intervals, when, according to the concept of fluid redistribution, a significant change in the stress - strain state of rock masses occurs.

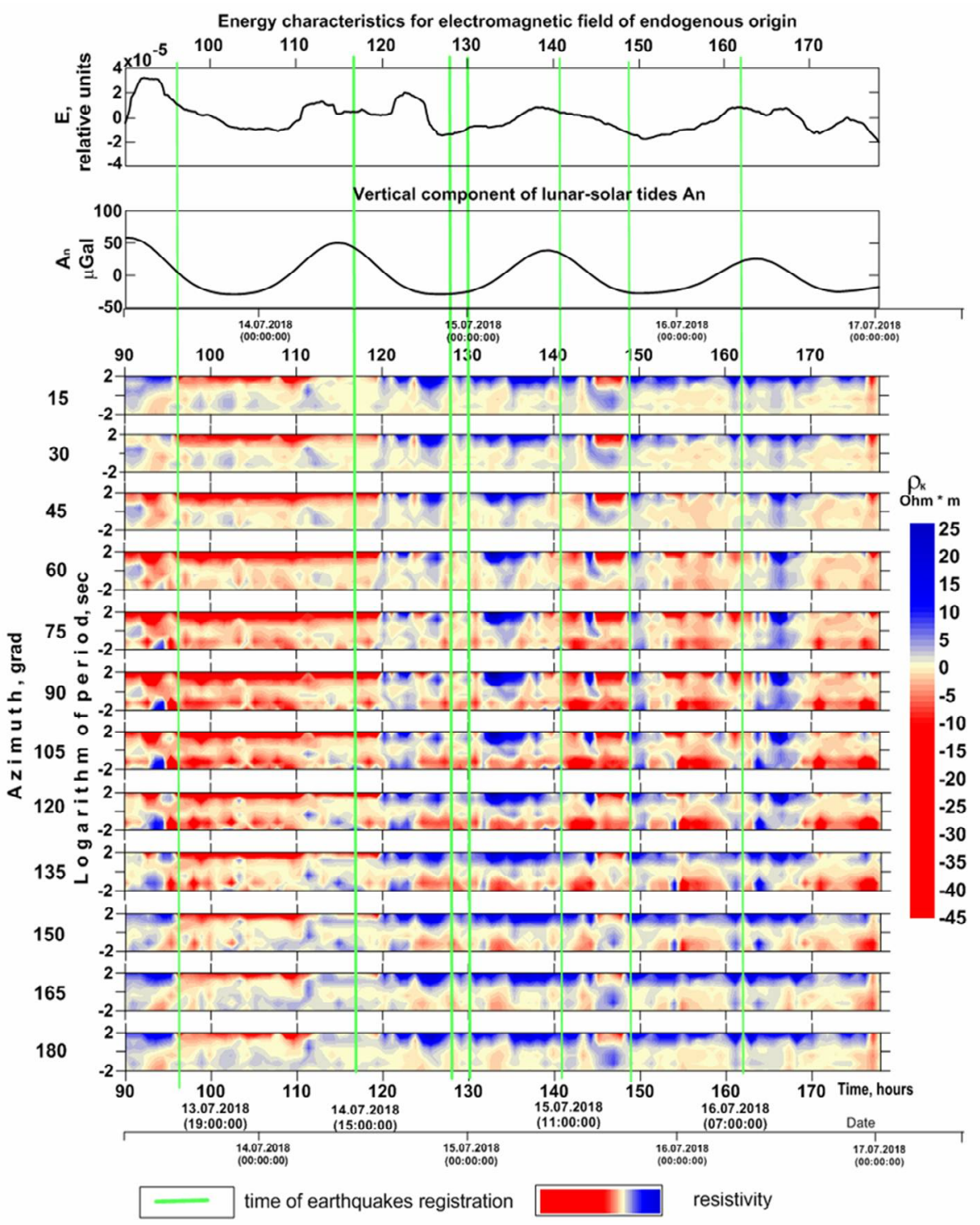

Fig. 7. Time-frequency series of azimuthal MT monitoring of apparent resistivity variations recorded during a field experiment (2018) at the Ukok MT monitoring site in the Kochkor Depression (Fig. 1). The green lines indicate earthquakes (Fig. 1).

This work was carried out in the framework of the RFBR project 17-05-00654 and the State Assignment of the Research Station RAS (No. 0155-2018-0001). 


\section{References}

1. E.A. Bataleva, V.Y. Batalev, A.K. Rybin Izv. Phys. of the Solid Earth, 49(3), 402 (2013)

2. E.A. Bataleva, Doklady Earth Sciences, 468(1), 523 (2016)

3. E.Yu. Ermolin, Scientific Reports on Resource Issues, 207 (2010)

4. A.A. Zhamaletdinov, F.P. Mitrofanov, A.D. Tokarev, A.N. Shevtsov, Doklady Earth Sciences, 371 (2), 403 (2000)

5. A.K. Saraev, M.I. Pertel, Z.M. Malkin, J. Appl. Geophys., 8(1-2), (2002)

6. Yu.A. Kugaenko Izv. Phys. of the Solid Earth, 41(7), 571 (2005)

7. L. Bogomolov, V. Bragin, A. Fridman, V. Makarov, G Sobolev., E. Polyachenko, G. Schelochkov, Zeigarnik V., A. Zubovich Comparative analysis of GPS, seismic and electromagnetic data on the Central Tien Shan TerritoryTectonophys., 431, 143 (2007)

8. Rybin A.K., Batalev V.Y., Bataleva E.A., Bragin V.D., Schelochkov G.G., Leonov M.G., Przhiyalgovskii E.S., Morozov Y.A. Doklady Earth Sciences, 470(1), 968 (2016)

9. A.A. Spivak Izv. Phys. of the Solid Earth, 46(4), 327 (2010) 